\title{
Design of Cherry Greenhouse Temperature Control System Based on CAN Bus
}

\author{
Anmin Wang ${ }^{1, a}$ and Shichao Liu $^{2, b^{*}}$ \\ 1Qingdao University of Science \& Technology, Qingdao, China \\ ${ }^{2}$ Qingdao University of Science \& Technology, Qingdao, China \\ a1049775677@qq.com, b927122707@qq.com \\ *The Corresponding author
}

Keywords: CAN bus; STM32, Pt100; Temperature control

\begin{abstract}
The application of CAN bus in the greenhouse temperature control in cherry is introduced. The design of STM32 based controller, two wire Pt100 temperature acquisition thermistor bridge measurement circuit through the output differential signal, amplifying the information by differential amplifier LM324, through the CAN bus transmission of analog signal, digital temperature the information through the STM32 comes with the A/D module. According to the actual temperature in the Greenhouse Cherry control requirements to control the ventilation device operation, finally realize the effective control of temperature in the Greenhouse Cherry.
\end{abstract}

\section{Introduction}

Cherry in greenhouse planting to control the temperature higher, the temperature sensor and the ventilation device in the temperature control process in plastic greenhouse needs more arrangement, CAN bus control circuit so the less connected to control the temperature in the greenhouse cherry. Controller Area Network (CAN) has the advantages of high communication speed and long communication distance, especially the ability to connect multiple unit nodes[1]. It improves the efficiency of the control system and reduces the management cost. In recent years, CAN bus control mode have been more and more widely used. Therefore, this paper introduces a temperature control system of Greenhouse Cherry STM32 and based on CAN bus, through the host computer to control the lower machine to achieve the monitoring of the temperature, and the temperature control target to control the ventilation device, then realize the temperature in the Greenhouse Cherry dynamic balance, provide a suitable temperature for cherry the growth of.

\section{Overall Design of Temperature Control System}

The main block diagram of the temperature control system is shown in Fig 1. It is mainly composed of the host computer, the RS232 serial module, the STM32 master controller, the CAN transceiver, the control node and so on. The main function of the control node is to collect the temperature signals from the Pt100 temperature sensor and upload it to the host computer, and finally control the operation of the ventilation device according to the needs of temperature control, and achieve the control objectives. The hardware of the temperature control system adopts the STM32 controller with CAN function, the control mode of CAN transceiver, the control mode has less control port, the hardware circuit connection is simple, and the software program is convenient to call. In the temperature control of cherry in greenhouse, shed every point will have a ventilation ventilation device and a corresponding temperature sensor. The main control objective control system is to detect the ventilation shed point temperature, real-time display on the display, and according to the temperature control requirements, control the ventilation device to maintain a stable temperature action. The STM32 master controller selects the microprocessor of the 32 bit STM32F103 enhancement type ARM core produced by stmicroelectronics. The STM processor itself features a CAN controller that can be directly connected to the CAN transceiver[2]. In addition, the processor also has 2 road 12 bit A/D conversion modules, each A/D module has 16 external channels, fully 
meet the needs of temperature information collection.

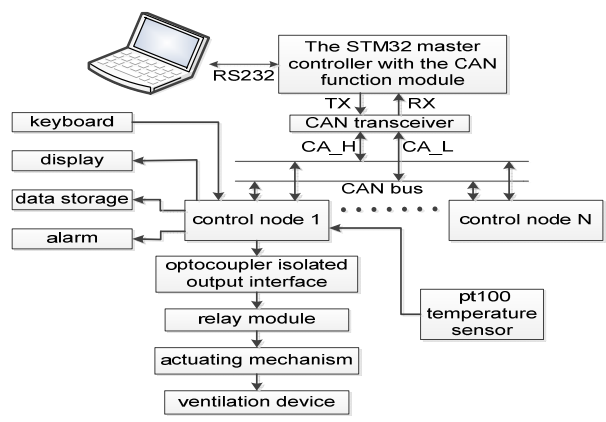

Figure 1. General block diagram of temperature control system

The Working Principle of CAN Controller. The CAN controller is used to convert the information to be transmitted to the CAN frame that conforms to the CAN specification, and to exchange information over the CAN bus via the CAN transceiver, as shown in Fig 2.

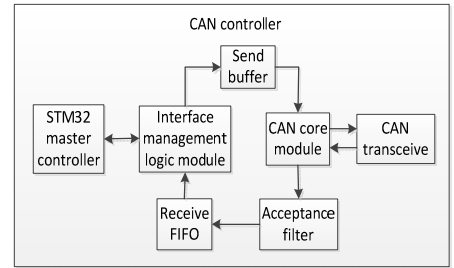

Figure 2. Working principle diagram of CAN controller

The temperature control of CAN protocol controller integrated in STM32 controller, the controller area network, with CAN 2.0A and 2.0B (active) technical specification, can send and receive data frame standard and remote frame, bit rate up to $1 \mathrm{M} / \mathrm{S}$, with its own carrying 2 approval and 14 Level 3 is adjustable register acceptance filter can filter out unwanted information, and reduces the workload of the main controller. Its internal SRAM buffer can handle up to 32 message messages. Whether the CAN controller is integrated on the STM32 controller or independent, its main components are composed of the interface management logic module, the CAN core module, the transmit buffer, the acceptance filter and the receiving FIFO. The main function of CAN core module is to realize the conversion between serial data and parallel data when accepting and transmitting information. When receiving the information, the CAN core module converts the serial data into parallel data[3]. When the information is sent, the parallel data is transformed into serial data, and the specific data transmission mode is shown in Fig 3.

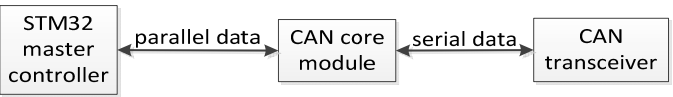

Figure 3. Block diagram of serial data and parallel data

CAN Transceiver. As shown in Fig 4, this design uses CAN PCA82C250 as the CAN transceiver driver interface controller and the physical bus ask, its main role is to be CAN controller logic level information into difference level information, provide differential transmit capability to the bus and the CAN controller differential receiving ability, the speed up 1M/S, compatible with ISO11898 standard. Its slope control function enhances the EMC performance, and the preparation mode can reduce the power consumption of the network[4]. In the preparation mode, the network will be activated immediately once it detects that there is a message on the bus. PCA82C50 has strong anti-interference ability, and also has the functions of thermal protection and short circuit protection, and the number of nodes supported by it can reach more than 110 . 
Between the node controller and the CAN bus transceiver, 2 high-speed optocoupler 6N137 are used for electrical isolation, which prevents bus interference from being introduced into the system and improves the reliability of the system. At the same time, one 120 terminal matching resistors are connected at the end of the node to improve the anti-interference ability of the data communication[5].

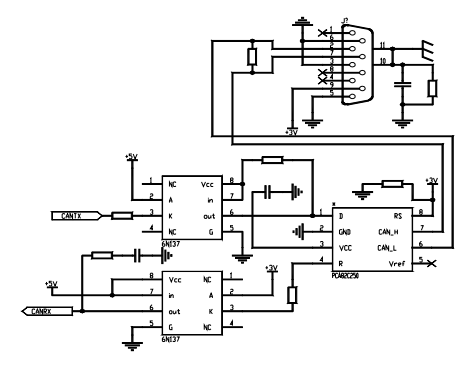

Figure 4. CAN node hardware circuit

\section{Temperature Collection in Cherry Greenhouse}

Selection of Temperature Sensors. Greenhouse Cherry for temperature control has very strict requirements, from flowering to final, mature, each stage for temperature requirements are different. The temperature range of access to relevant information in roughly between -20 to 30 degree, comprehensive consideration of various factors of choice of temperature measurement accuracy, stability and reliability of the positive temperature coefficient of Pt100 platinum resistance, its performance fully meet the needs of greenhouse temperature acquisition of cherry.

The platinum resistance can obtain very high measurement accuracy in the range of medium temperature -50 degree to 600 degree. The platinum resistance $\mathrm{R}$ varies with the temperature, and the functional relationship between the platinum resistance value $\mathrm{R}$ and the temperature $\mathrm{t}$ is expressed by the following equation[6]:

$$
\begin{aligned}
& R_{t}=R_{0}\left(1+A t+B t^{2}\right), \quad 0^{\circ} \mathrm{C} \leq t \leq 600^{\circ} \mathrm{C} \\
& R_{t}=R_{0}\left[1+A t+B t^{2}+C(t-100) t^{3}\right],-50^{\circ} \mathrm{C} \leq \mathrm{t} \leq 0^{\circ} \mathrm{C}
\end{aligned}
$$

$\mathrm{Rt}, \mathrm{Rt}-$ Resistance values of platinum resistance at temperatures of $\mathrm{t}$ and 0 degrees;

$\mathrm{A}, \mathrm{B}, \mathrm{C}$ - The constants measured by experiments, in which

$$
A=3.96847 \times 10^{-3}\left(1 /{ }^{\circ} \mathrm{C}\right) \quad B=-5.847 \times 10^{-7}\left(1 /{ }^{\circ} \mathrm{C}\right) \quad C=-4.22 \times 10^{-12}\left(1 /{ }^{\circ} \mathrm{C}\right)
$$

Acquisition and Amplification of Temperature Signals. This paper finally selected the two wire system Pt100 platinum resistance, using the bridge temperature measuring method, the specific temperature measurement method and circuit diagram, as shown in Fig 5.

Design of constant voltage source circuit for temperature sensor. Temperature measurement scheme chose bridge measurement circuit, in order to work with the $\mathrm{R}_{\mathrm{pt100}}$ and $\mathrm{R}_{3}$ the stability of resistance of $R_{2}$ and $\mathrm{RX}_{2}$ between the bias voltage, the positive supply reference of the bridge must be stable, because when the Vcc voltage fluctuates, the voltage signal behind the amplifier output will change, and the temperature thermistor parameters stable based on the Vcc network will no longer be accurate. Therefore, a TL431 controllable precise voltage stabilizing source is arranged between the voltage source Vcc and the bridge temperature measuring circuit. The TL431 can set any voltage value from its reference voltage $\mathrm{V}_{\text {ref }}(2.5 \mathrm{~V})$ to $36 \mathrm{~V}$ by adjusting its external fixed resistance $\mathrm{R}_{1}$ and bias resistor $\mathrm{RX}_{1}$. Under the function of the voltage regulator TL431, the power Vcc stabilizes the $4.1 \mathrm{~V}$ voltage required by the bridge type temperature measuring circuit, and then improves the accuracy of the Pt100 temperature measurement. After the TL431 voltage regulator, the output voltage calculation formula is shown as follows[7]:

$$
\begin{aligned}
& U_{o}=V_{\text {ref }}\left(1+\left(R_{1} / R X_{1}\right)\right) \\
& \mathrm{U}_{\mathrm{O}}-\mathrm{TL} 431 \text { regulator output voltage } \\
& \mathrm{V}_{\text {ref }}-\mathrm{TL} 431 \text { internal reference voltage }(2.5 \mathrm{~V})
\end{aligned}
$$


$\mathrm{R}_{1}$-Fixed resistance

$\mathrm{RX}_{1}$ - Bias rheostat

The constant voltage source circuit composed of TL431 finally provides a stable power supply of 4.096V for our bridge temperature measuring circuit. The bridge temperature measuring circuit is essentially bridge voltage sampling circuit. As shown in Fig. $5, R_{2}, R_{3}, R_{p t 100}$ and $R_{2}$ constitute the measuring bridge, set $R_{2}=R_{3}$, adjust the resistance value of $R_{2}$ and $R_{3}$ with the same amplitude, and can adjust the magnitude of the output differential signal $V_{1}$ and $V_{2}$. A precise adjustable resistor $\mathrm{RX}_{2}$ is placed on one of the bridge arms, which can be used to adjust the magnitude of the differential voltage input to the operational amplifier, and to adjust the zero temperature. Only a few $\mathrm{V}_{2}$ differential signal that signals are generated between the bridge temperature measuring circuits $\mathrm{RX}_{2}, \mathrm{~V}_{1}$. Through the $-5 \mathrm{~V}$ power operated single operational amplifier differential amplifier LM324AD, the differential mode signal is amplified to the voltage required by the AD conversion chip.In differential amplifier circuit $\mathrm{R}_{5}=\mathrm{R}_{6}, \mathrm{R}_{7}$ and the reverse input feedback resistor grounding resistance is equal to $R_{4}$, in theory, Single operational amplifier differential amplifier output voltage signal for amplification $R_{7} / R_{6}$ by differential mode signal voltage of $\left(V_{1}-V_{2}\right)[8]$, so the difference of single operational amplifier input and output amplifier of the calculation formula is as follows:

$$
V_{\text {out }}=R_{7}\left(V_{1}-V_{2}\right) / R_{6}
$$

Of these, $R_{7} / R_{6}$ determines the amplification capability of the differential signal $\left(V_{1}-V_{2}\right)$.The greater the ratio of $R_{7} / R_{6}$ or the greater the differential signal $\left(V_{1}-V_{2}\right)$. The differential amplifier output voltage gain is also bigger. Therefore, by changing the ratio of $R_{7} / R_{6}$, it can meet the temperature requirements set by the cherry greenhouse.

Output formula of differential mode signal[9]:

$$
V_{1}-V_{2}=4.096\left(\frac{R_{p t 100}}{R_{2}+R_{p t 100}}\right)-\frac{R X_{2}}{R_{3}+R X_{2}}
$$

Through a single operational amplifier, differential amplifier can not only amplify the differential mode signal, but also suppress the interference of common mode signal, and can also improve the accuracy of temperature measurement.

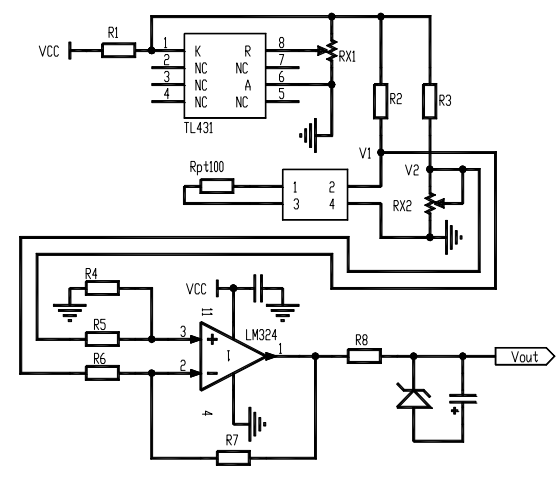

Figure 5. A circuit diagram of a signal amplifying circuit

\section{Real Time Control of Temperature}

The main function of the CAN control node is using node controller with A/D transducer bridge measurement circuit output differential voltage obtained by calculating the node temperature, the temperature information is transferred to the STM32 main controller through CAN bus.According to the actual needs of temperature control, the main controller transmits the control instructions to the control node through the CAN bus, and the control node controls the ventilation device to achieve the control goal[10].The temperature control flow chart is shown in Fig 6. 


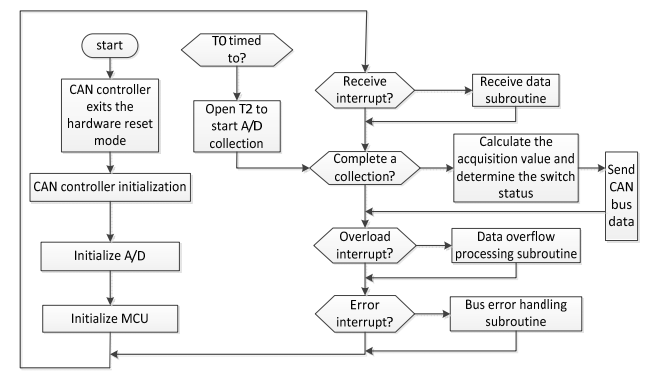

Figure 6. Flow chart of temperature control

\section{Summary}

The design of the temperature control system of STM32 control of effective control of cherry in Greenhouse Based on temperature, has a very high social impact and economic benefits, reduce the work intensity of the staff, has a wide application prospect. The transfer of information based on CAN bus improves the accuracy of information transfer.STM32 price is cheap, control effect is good, operation speed is fast. Two wire Pt100 thermistor wires due to the presence of resistance may lead to measurement error, considering the application of the temperature control precision is not particularly high, so the two wire Pt100 fully meet the requirements of temperature control of greenhouse cherry.

\section{References}

[1] P.Liu: Design and implementation of dual CAN bus based on STM32F407 [J]. Technology Vision,2016,(13):130+132. (In Chinese)

[2] G.X. Tong, L.Fu and H.Liu: Application programming and implementation of STM32 based on CAN bus[J].Information Technology,2015,(10):49-52. (In Chinese)

[3] L.X.Luan:Design of temperature measurement controller based on STM32[J].Industrial Technology Innovation,2015,(01):39-42. (In Chinese)

[4] H.B.Ai, J.H.Wei, Q.Qiu and W.G.Zheng: Design of temperature and humidity monitoring system for miniature plant based on STM32 [J].Agricultural Mechanization Research, 2014,(05):141-144+150.(In Chinese)

[5] J.H.Tang: CAN bus analysis and application programming[D].University Of The Inner Mongol, 2013.(In Chinese)

[6] X.L.Kang: Design of CAN bus communication based on STM32[D].Jilin University,2013.(In Chinese)

[7] T.Su: Design of temperature control system for group control instrument based on STM32 and CAN bus[D].Electronic University Of Science \&Technology Of Hangzhou,2013.(In Chinese)

[8] H.G.Sui: Application of PT100 temperature sensor in real time monitoring system of temperature data[J].Computer development and Application,2011,(04):64-65.(In Chinese)

[9] Y.Q.Liu,J.Zhao: Temperature monitoring system based on STM32 and CAN bus[J].Electronic Technology, 2010,(10):58-59.(In Chinese)

[10]M.Hou, Y.Du:Design of interface circuit based on CAN bus[J]. Communications technology, 2008,(07):138-140. (In Chinese) 